\title{
THE HOLIFIELD HEAVY-ION RESEARCH FACILITY AT OAK RIDGE
}

J. B. Ball, J. A. Martin, J. A., Biggerstaff, C.' M. Jones,

R. S. Lord and R. L. Robinson

(Paper presented by F. Plasil)

Oak Ridge National Laboratory,* Oak Ridge, Tennessee 37830, USA

Abstract. This paper describes the new accelerator facility under construction at Oak Ridge.

Résumé. Cet article décrit le nouvel accélérateur et laboratoire en construction à Oak Ridge.

At Oak Ridge we are now well into the first phase of a major expansion of our heavy ion accelerator facilities. This present phase, begun in late 1974, will include a 25 MV tandem electrostatic accelerator, a building to house the tandem with some additional experimental areas for use with the ion beams directly from the tandem, equipment to transport heavy-ion beams from the tandem to the existing Oak Ridge Isochronous Cyclotron (ORIC), and necessary modifications to ORIC so that these beams may be injected and further accelerated.

Figure 1 illustrates the ion energy performance as a function of ion mass for heavy ion beams available present1y from ORIC, those expected from the $25 \mathrm{MV}$ tandem accelerator, and those expected from the tandem + ORIC combination. Both the tandem and tandem + ORIC curves assume two strippings. The tandem curve is computed for a gas stripper in the terminal and foil stripper in the

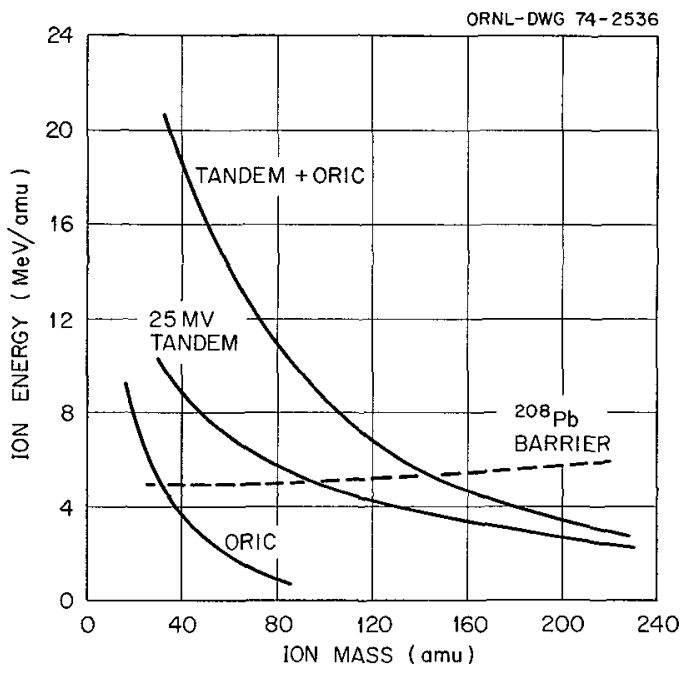

Fig. 1. Beam energy as a function of ion mass for the separate and combined accelerators. high-energy accelerating tube. The tandem + ORIC curve is computed for gas stripping in the terminal and foil stripping in ORIC. Both cases assume selection of the most probable charge state following each stripping so that both curves could be raised slightly (at the cost of intensity) by going off the peak of the charge state distribution.

The dashed line marks the position of the Coulomb barrier for any of these ions on a lead target nucleus. Rough1y speaking, to study processes that involve nuclear reactions we need to have an ion with energy greater than (above) this line. Thus for lons heavier than about $A=95$ such studies will require using the ORIC to boost the energy of the tandem beams.

When not operating in the coupled mode, the two accelerators will operate independently supporting two experimental groups.

The total cost of the present project will be about $17 \frac{1}{2}$ milition dollars and completion is scheduled for May 1979.

1. Tandem Accelerator.

The $25 \mathrm{MV}$ electrostatic accelerator is being procured commercially from National Electrostatics Corporation. The design and fabrication of the accelerator system will be provided by NEC followIng detailed technical specifications developed by the Oak Ridge group.

The most novel feature of the new tandem accelerator is its folded configuration. In this accelerator, both "low-energy" and "high-energy" acceleration tubes are contained within a single column. Negative ions are injected into the lowenergy acceleration tube and accelerated to the

*Operated by Union Carbide Corporation for the U.S. Energy Research and Development Administration. 
high voltage terminal which is maintained at positive potential. In the terminal, the ion beam first passes through a stripper and becomes positively charged. After stripping, one charge state component present in the beam is bent by a magnet through angle of $180^{\circ}$ and infected into the high-energy acceleration tube for further acceleration back to ground potential. The essential polnt is that a folded tandem accelerator requires only one column structure in contrast to a conventional or "1ineer" tandem accelerator which employs two column structures, one on each side of the high voltage terminal.

The basic layout of the accelerator is shown schematically in Fig. 2 and performance parameters are listed in Table 1.

The foundation of any electrostatic accelerator is its column structure. In this machine, the column will be built in 24 inch high modules consisting of 4 inch thisk horizontal cast aluminum bulkheads supported by 16 c1reumferentially located column posts of the type used in previous NEC designs. The eolumn structure will have a diameter of 11 feet and a leng of 62 feet, excluding the high voltage terminal. The high yoltage terminal will have a diameter of 13.3 feet and a helght of 16 feet.

The acceleration tubes will be the standard NEC design used in virtually all of their previous accelerators. These are fabricated of titanium and alumina ceramic bonded together with an all-metal seal. They utilize flat, field electrodes and are designed to operate at ultramigh vacuum. Voltage grading for each acceleration tube and the column structure will be provided by three enclosed corona discharge tubes,

Two independent groups of three pelletron chains will be used for charge transpont to the high voltage terminal. These systems together will deliver current of $600 \mu \mathrm{A}$. Provision has also been made for future installation of an additional group of three chains to waise the total charging capability to $900 \mu \mathrm{A}$. Power for various components within the column structure will be provided by two rotating shafts, each capable of transmitting 50 horsepower.

Service for the colum structure and contents of the high voltage terminal will be performed from two movable service platforms. The large annular platform will be located in the space between the column and pressure vessel. A small platform ca11ed the "column service platform" will move vertica1ly wthin the column allowing easy access to the Interior of the column structure and high voltage terminal.

The accelerator will be insulated by pure $\mathrm{SF}_{6}$ gas contained within a pressure vessel 98 feet long and 33 feet in diameter. This vessel, with a maximum design pressure of $110 \mathrm{psig}$, will welgh approximately 438 tons empty and approximately 500 tons when the column structure and associated equipment is installed.

Another signifteant break with the tradition of electrostatic accelerators fs with the choice of a control system: our system will be based entirely on digital communication techniques. Although digital computers will play a very important role in the control system, strictly speaking we are not going to have a "computer control system." That iss, we do not plan to have the computers Inside any of the eontrol 1oops of the accelerator performing control functions without operator intervention. We believe that conventional analog servo loops (e.g., "corona stabilizer") will perform satisfactorily with only minor changes.

Initially, the task of the digital elements of the control system will be to provide multiplexed communication of the information between the operator and the accelerator. This will greatly reduce the amount of signal wiring required and will facilitate implementation of the two identical control consoles required (one for use with ORIC and one when the tandem is used by itself). Although not computer controlled, the accelerator will be computer controlleb1e. Brovtsions have peen incorporated to insure, as we learn how the accelerator behaves, that we can gradualiy teach the computex to take over some of the human opexators' tasks.

\section{Building.}

The new building for the tandem accelerator adjoins the present ORIC building. It consists of a three-story addition with a tower housing the tandem pressure vessel. The tower will have a diameter of approximately 45 feet and will rise 160 feet above ground. A sectional view is shown in Fig. 3, The shielding, ranging up to four feet of concrete, provides adequate protection for $\sim 1$ puA of beams with $A>4$ at all energies.

A small, $40 \mathrm{ft}$ by $53 \mathrm{ft}$, two-story structure located nearby will house the $\mathrm{SF}_{6}$ gas storage tanks and compressors for gas transfer. 
Table 1. Tandem Performance Parameters

$\begin{array}{ll}\text { Ion Mass: } & 12 \text { to } 250 \\ \text { Analyzed Beam Intensity: } & 1.0 \mathrm{p \mu A} \\ \text { Maximum Analyzed Beam Emittance } & \sim 0.4\left[1+(\mathrm{q}+1)^{1 / 2}\right] /(1+\mathrm{q})^{1 / 2} \pi \mathrm{cm} \mathrm{mrad} \\ \text { Operating Terminal Potential: } & 7.5 \text { to } 25.0 \mathrm{MV} \\ \text { Typical Beam Energy Stability: } & \sim \pm 2 \mathrm{keV} \mathrm{x} \mathrm{accelerated} \mathrm{ion} \mathrm{charge} \mathrm{number} \\ \text { Injected Ion Energy: } & 150-500 \mathrm{keV} \\ \text { Injector Magnet: } & 120 \\ \text { a) mE/q } & \sim 1 / 250 \text { (fwhm) } \\ \text { b) minimum mass resolution } & 320 \\ \text { Analyzing Magnet: } & \sim 1 / 1000 \text { (fwhm) } \\ \text { a) mE/q } & \\ \text { b) minimum energy resolution } & \end{array}$

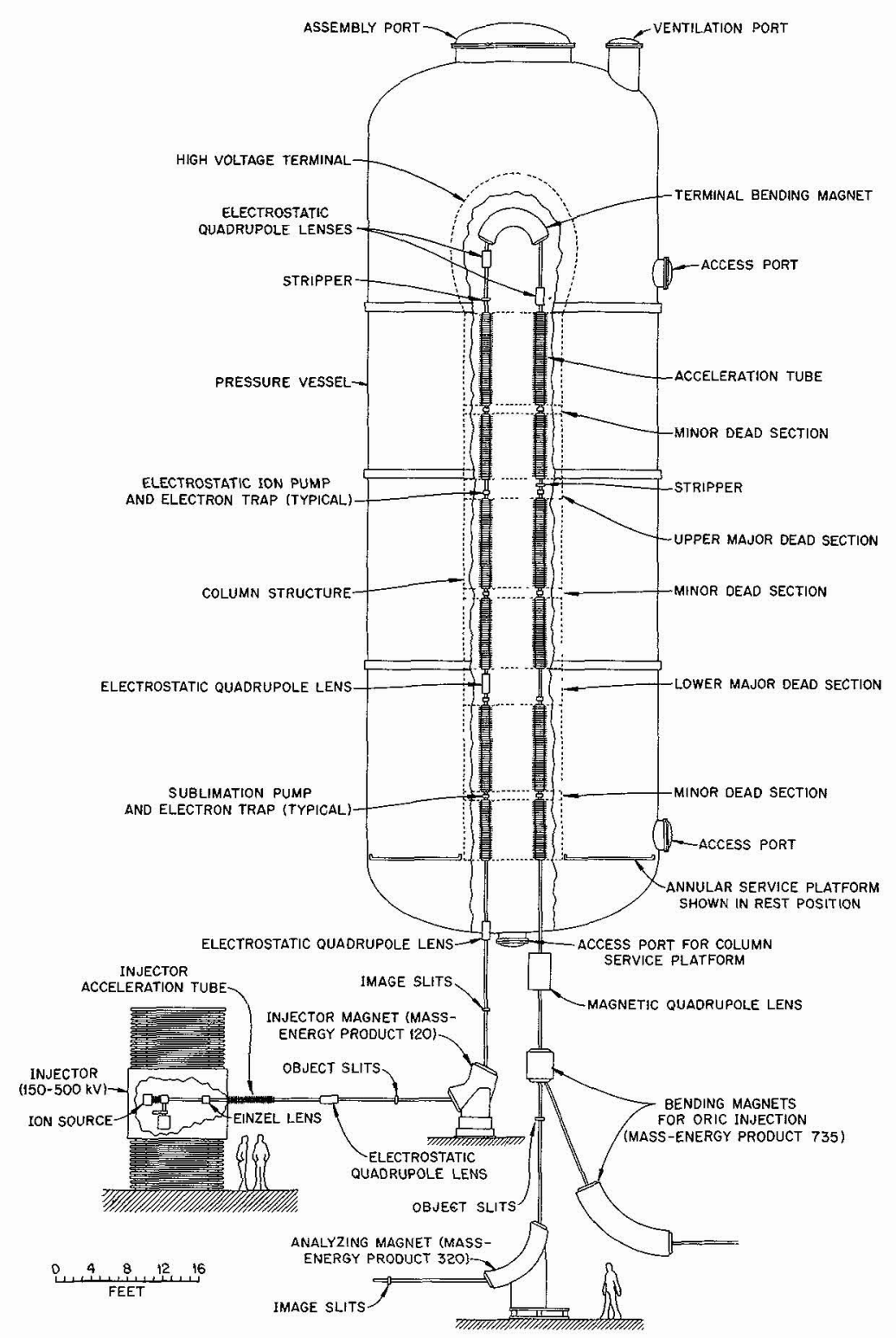

Fig. 2. A schematic view of the $25 \mathrm{MeV}$ tandem accelerator system 


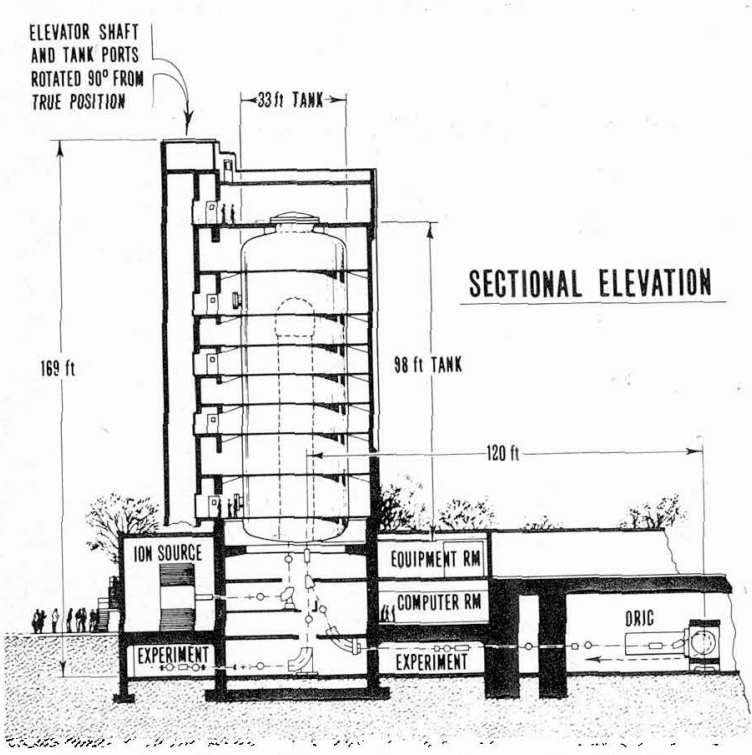

Fig. 3. Elevation view of new faclilty

The post-accelerator beam is analyzed by one of two magnet systems. The first, consisting of fixed $25^{\circ}$ and $65^{\circ}$ magnets wi.th $\rho=10 \mathrm{ft}$, is for injecting the beam into the ORIC cyclotron. The mass energy product of 735 was chosen to bend any tandem beam with charge state near the equilibrium charge state as produced by a gas stripper at the tandem terminal. The second system is a single $90^{\circ}, p=$ 63 in. magnet on a rotatable mount that is used to inject the beam into any beam line in two new experimental areas. This magnet with mass energy product of 320 will bend any beam from the tandem that has sufficient energy for nuclear physics studies.

Figure 4 shows the total experimental facility for both machines including the existing beam lines. The existing lines are used with ORIC alone or with the tandem and ORIC coupled. The location of eight proposed new beam lines are shown emanating from the tower base.

\section{ORIC Modifications.}

To further enhance the research capabilities of heavy ion beams produced by the tandem, the existing cyclotron, ORIC, will be suitably modified to serve as an energy booster. Thus, lons accelerated in the tandem will be transported to the ORIC, injected into the center of the cyclotron and then accelerated to full energy.

As shown in Fig. 5, the beam will enter the cyclotron through the dee stem, and be directed by an inflection magnet so that after entering the

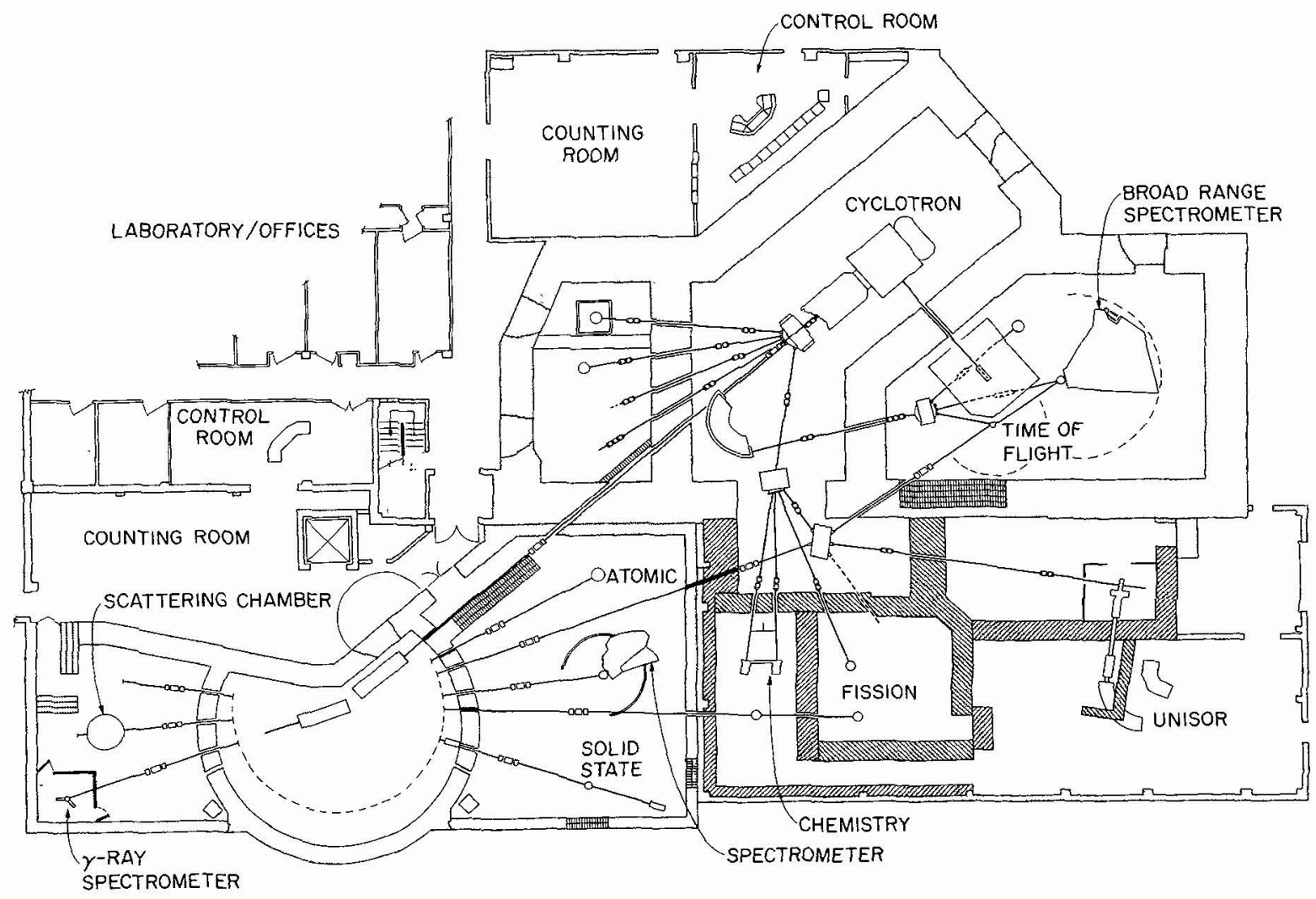

Fig. 4. Conceptual layout of Heavy Ion Laboratory beam lines and expeximental equipment 


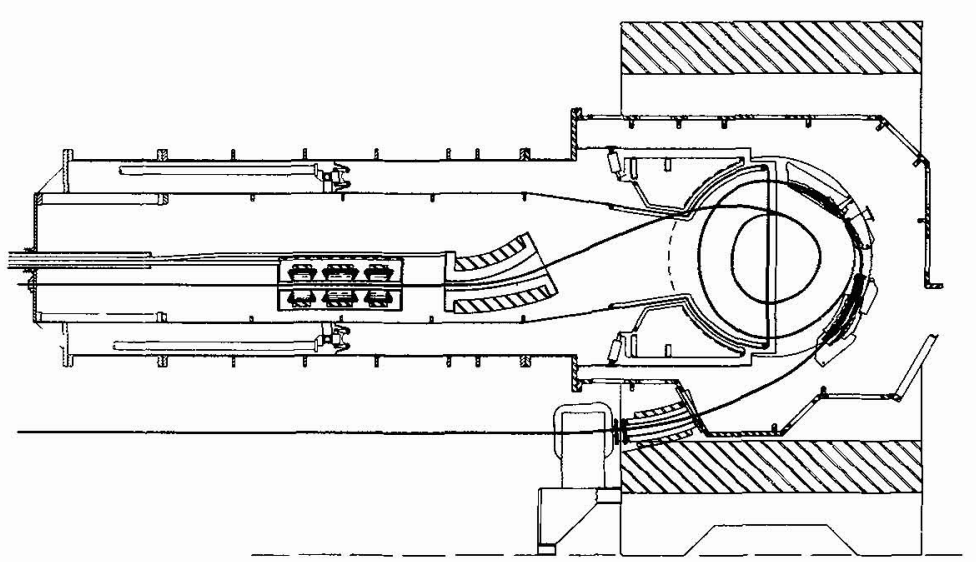

Fig. 5. The injection of an ${ }^{127}$ I beam into ORIC

magnetic field it is tangent to an orbit suitable for acceleration. A foil is located at the point of tangency to strip the beam to a higher charge state so that it can be contained in the magnetic field. After stripping, typically about $20 \%$ of the beam will be in the appropriate charge state for acceleration. In the figure a beam of $225 \mathrm{MeV}$ ${ }^{127} \mathrm{I}^{8^{+}}$stripping to $32^{+}$and being accelerated to $725 \mathrm{MeV}$ is used as an example.

The foil positioner will be designed to accommodate a "magazine" of several foils so that new foils can be inserted into the beam essentially instantaneous $1 \mathrm{y}$ without breaking vacuum or altering the position of the foil holder.

\section{Phase II Booster Accelerator.}

While construction is progressing on the present facility, we have been actively pursuing a design study for a Phase II booster capable of accelerating all ions to energies in excess of the Coulomb barrier.

Our Phase II proposal is based on a separated sector cyclotron with an energy constant of 400 . The separated sector machine would be located so that either the $25 \mathrm{MV}$ tandem or the ORIC could serve as the injector. The energy performance of this booster accelerator injected with the 25. MV tandem is compared with various accelerators in Fig. 5. The time scale for construction is such that, if funded in 1978, the accelerator would be operational in early 1982.

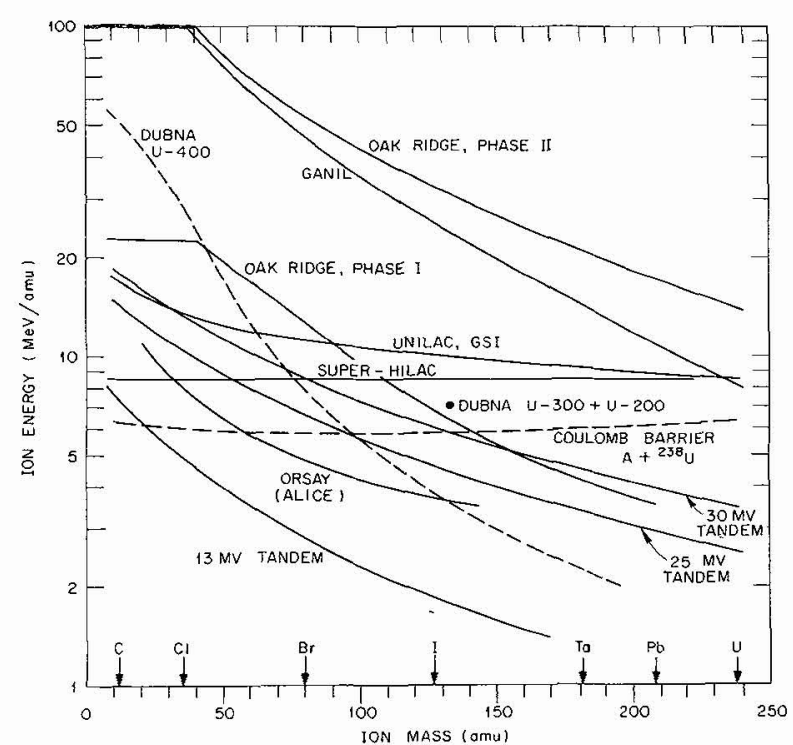

Fig. 6. Ion energy capabilities for various accelerators 